\title{
A Critical Overview of Internal and External Cylinder Contact Force Models
}

\author{
Cândida M. Pereira ${ }^{1}$, Amílcar L. Ramalho ${ }^{2}$ and Jorge A. Ambrósio ${ }^{3}$ \\ ${ }^{1}$ Polytechnic Institute of Coimbra \\ R. Pedro Nunes, 3030-199 Coimbra, Portugal \\ e-mail: candida@isec.pt \\ ${ }^{2}$ University of Coimbra \\ R. Luís Reis dos Santos, 3030-788 Coimbra, Portugal \\ e-mail: amilcar.ramalho@dem.uc.pt \\ ${ }^{3}$ Technical University of Lisbon \\ Av. Rovisco Pais, 1049-001 Lisboa, Portugal \\ e-mail: jorge @dem.ist.utl.pt
}

\begin{abstract}
Most of the analytical models found in the literature, to study the contact between cylindrical bodies, are based on the Hertz pressure distribution. The major shortcomings associated with these cylindrical models concerns their nonlinearity. Firstly, the indentation is expressed as an implicit function of the contact force, thus a numerical iterative technique is required to evaluate the contact force for a given indentation. In a dynamic analysis code this implies that at each integration time step, the iterative process for the solution of the nonlinear equations has to be solved. Secondly, the current cylindrical contact models include logarithmic functions, which impose mathematical and physical limitations on their application, particularly for conformal contact conditions with lower clearance values. The validity domain of each contact model is identified in this work with relation to the clearance value and material properties of the contacting cylinders. A comparative assessment of the performance of each model is performed calculating the relative difference of each one in relation to Johnson's model. The results show that, in general, different models exhibit distinct behavior for both the internal and external contact between cylinders. The load limit of each model and the restrictions on its application is identified using two simple examples of mechanical engineering practice in which internal contacting cylinders are involved are analyzed: journal bearings and roller chain drives.
\end{abstract}




\section{Introduction}

The analytical models available to study the contact stresses and deformations are important design tools for the kinematics of mechanical systems or in tribology studies and therefore their extensive use has to done within their validity limits. Several models have been postulated to represent the interaction forces between the surfaces of two contacting bodies. The simplest contact description, known as the Kelvin-Voigt viscoelastic model, represents the contact/impact force by a parallel linear spring-damper element [1]. Given its simplicity, this model is a very rough approximation and does not represent accurately the overall nonlinear nature of the contact phenomenon. Firstly, it is very difficult to quantify the stiffness coefficient, which depends on the geometry and physical properties of the contacting bodies. Secondly, it is an oversimplification to assume a linear relation between the indentation depth and the contact force; the contact force depends on the shape of the surfaces in contact, the material properties, and so forth. Thus a more complex relationship between indentation, i.e., the pseudo-penetration between the contacting bodies, and the contact forces is generally required [2].

The impact response can be accurately predicted using the traveling stress wave propagation theory. However, this analysis is quite complicated [3]. A more suitable model that expresses the nonlinear relationship between the impact force and the indentation depth is the non linear force-displacement model by Hertz [4, 5]. This is an elastostatic theory that does not account for the energy dissipation process that characterizes impact mechanisms. As a result, the Hertz contact model cannot be used during the loading and unloading phases of contact unless this is quasi-static and for very well defined geometries. This issue has been investigated by Lim and Stronge [6] and by Gugan [7] that showed that the compression part of the impact is well described by the Hertz theory for ball and cylindrical geometries but that, even for elastic contact only, during restitution a non negligible amount of energy is lost, mainly due to the elastic strain energy that develop. During dynamic contact, or impact, the loss of energy depends on the relative size of the bodies, their materials and the relative impact velocity. These drawbacks led several researchers to modify the Hertz law to include energy dissipation in the form of internal damping [8-10], being the model proposed by Lankarani and Nikravesh an example that is extensively used. Another problem of the Hertz contact model is that the compression of two-dimensional bodies in contact cannot be solely calculated from the contact stresses given by the Hertz theory. The shape and size of the bodies and the way in which they are supported must be taken into account also. These calculations are generally difficult, 
particularly if the elastic compression of the bodies is a line instead of a point. One of the major difficulties is the identification of suitable influence functions that take into account variations in cylindrical contact. Several analytical models are proposed to calculate the indentation of cylindrical surfaces and the contact force exerted between them when the contact area is rectangular $[5,9,12-14]$. However, three major drawbacks are associated with these models. First, the contact force cannot be explicitly defined as a function of indentation in a closed form $[2,15]$. This poses a problem when implementing forward dynamic analysis computational programs for impact simulation, because a numerical iterative technique is required to predict the contact model parameters, i.e., contact stiffness and damping, at each integration time step [16-18]. Furthermore, the control of the time steps of several numerical integration algorithms requires the information on any new contact indentation and the respective contact force that develops during the time-step in order to avoid excessive initial indentations to develop [19]. Secondly, being these models based on the Hertz pressure distribution it should not be taken for granted they suitable for applications where the size of contact area is comparable to the dimensions of the contacting bodies, i.e., in conformal contact conditions. It is well known that the analytical model derived by Hertz is only applicable in a limited range of conditions, notably in conditions of non-conformal contact, where the dimensions of the deformed contact patch are small in comparison with the principal radii of the undeformed surfaces $[4,5]$. The third drawback is related to the expressions that define all of these models, where a logarithmic function imposes some mathematical and physical limitations on internal contact analysis. Several new cylindrical contact models are being proposed, such as that by Liu and co-workers $[20,21]$ for internal cylindrical contact. Frequently the validation of these models is done by using a detailed finite element analysis [6, 20,21] for a range of clearances specific for the applications foreseen in each one of them. Consequently, each cylindrical contact model has a specific validity domain of application, which depends on the clearance value and the material properties.

The purpose of this paper is to present and discuss the shortcomings associated with the actual cylindrical contact models to describe the contact between bodies with cylindrical geometries with particular focus on internal contacting geometries involving low clearances and high loads simultaneously. Examples of the application of these models are the analysis of machinery [22], chain drives [23], road and rail vehicles [24], etc., in which standard mechanical construction tolerances apply. The accuracy of each model is analyzed considering a wide range of contact conditions. In all models studied here only 
the compression phase of the contact is analyzed being the restitution phase of a dynamic contact not focused here. For a detailed analysis of the energy dissipation issues associated to dynamic contact, or impact, the interested reader is referred to the works by Hunt and Crossley [8], Lankarani and Nikravesh [7], Lim and Stronge [6] or Gugan [7] among others.

\section{Analytical models for contacting cylindrical bodies modeling}

Most of the models available in the literature describing the contact between cylindrical geometries express the contact force as an implicit function of the indentation. As a result, when used in the framework of forward dynamic analysis, a numerical iterative technique is required to evaluate the contact force at each integration time step. This is not only computationally costly but also represents a numerical difficulty for the performance of a computational program [16-18].

An analytical model that explicitly defines the indentation as a function of the contact force is more efficient for implementation in a computational code for impact simulation of dynamical systems. Therefore, despite the model proposed by Lankarani and Nikravesh $[9,10]$ has been established for modeling the contact between spherical bodies it has been extensively used in cylindrical contact analysis also [2, 25-31]. For this reason, this model is also considered in the comparative study of analytical contact models that describe the contact between cylindrical geometries, presented in this section.

\subsection{Johnson Model}

Johnson presents a study based on the Hertz theory [32], about the compression of a long circular cylinder in a non conformal contact with two other surfaces [5]. This approach, considering only the contact between two cylindrical bodies, is schematically represented in Figure 1. When two deformable cylinders of radius $R_{i}$ and $R_{j}$ made of materials with elastic modulus and Poisson coefficients denoted by $E_{i}, v_{i}$ and $E_{j}, v_{j}$, respectively, are submitted to the action of a compressive load $P$ the total indentation, $\delta$ is given by

$$
\delta=\frac{\mathrm{P}}{\pi \mathrm{E}^{*}}\left\{\ln \left(\frac{4 \pi \mathrm{E}^{*} \Delta \mathrm{R}}{\mathrm{P}}\right)-1\right\}
$$

In equation (1) the compressive load $P$ is expressed per unit of the axial length of the cylinder. Furthermore, the indentation, which accounts for the contribution of both 
cylinders, is assumed to be measured at a point distant enough from the contact point. $E^{*}$ represents the composite modulus of the two colliding cylinders and is evaluated as

$$
\frac{1}{E^{*}}=\frac{1-v_{i}^{2}}{E_{i}}+\frac{1-v_{j}^{2}}{E_{j}}
$$

However, if the contacting cylinders are characterized by similar elastic properties (Poisson's coefficient and Young's modulus), equation (2) takes the form

$$
E^{*}=\frac{E}{2\left(1-v^{2}\right)}
$$

Depending on what parameter $\Delta R$ represents, equation (1) can be applied to internal and external contacts. When $\Delta R$ represents the sum of the cylinders' radii, $\left(R_{i}+R_{j}\right)$, an external contact geometry is considered, as in Figure 1. Otherwise, for internal contact, $\Delta R$ is quantified by the difference between the cylinders' radii, $\left(R_{i}-R_{j}\right)$, corresponding to the clearance between the two cylindrical bodies.

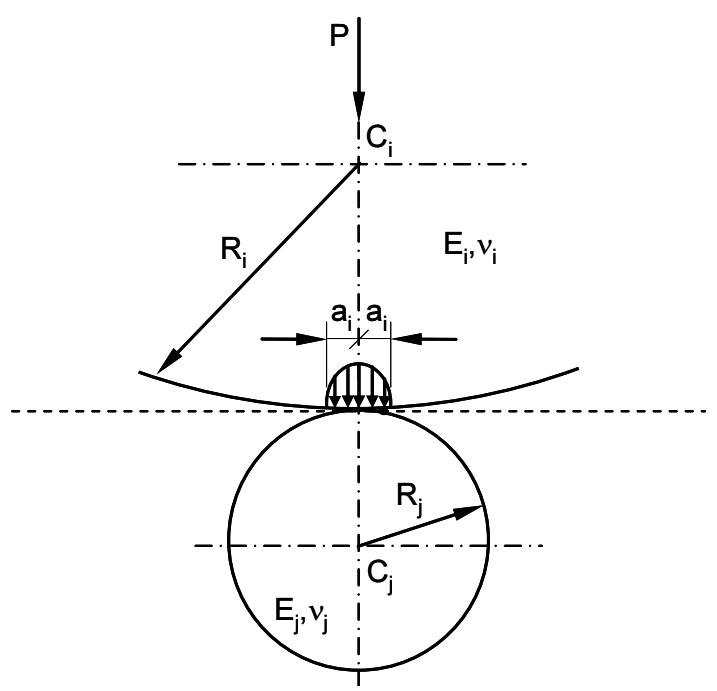

Figure 1 - Contact between cylindrical bodies [5].

In the numerical solution of the forward dynamics of a mechanical system the state variables, i.e., positions and velocities of the bodies in the system, are obtained first. The internal and external forces acting on the system are obtained with appropriate models, contact models in this case, with the information of the state variables. Therefore, the forces are calculated for given positions and velocities of the system at each time step. 
Therefore, for each given indentation, equation (1) has to be solved iteratively to evaluate the contact force that fulfills it [2]. Considering that the generalized stiffness depends on the contact force, namely due to the variation of $\Delta R$, a different stiffness value is obtained for each instant of contact. This is a difficulty when implementing a computational program for impact simulation analysis, because a numerical iterative technique is required to predict the contact parameters at each integration time step [16-18].

The ESDU - 78035 Tribology Series [11] contains some expressions for contact mechanics analysis suitable for engineering applications. For the particular case of contacting cylinders made of materials with identical elastic properties the model proposed by ESDU is equivalent to the one presented by Johnson [5].

\subsection{Radzimovsky Model}

Formulas for Stress \& Strain [33] includes a contact expression proposed by Radzimovsky [12] to calculate the reduction in the distance between centers for contact between external cylinders, i.e., the indentation. This expression is given by

$$
\delta=\frac{\mathrm{P}}{\pi \mathrm{E}^{*}}\left[\frac{2}{3}+\ln \left(\frac{2 \mathrm{D}_{\mathrm{i}}}{\mathrm{b}}\right)+\ln \left(\frac{2 \mathrm{D}_{\mathrm{j}}}{\mathrm{b}}\right)\right]
$$

where $D_{i}$ and $D_{j}$ represent the diameter of contacting cylinders. For cylinders with different elastic material properties, parameter $b$, appearing in equation (3), is evaluated by

$$
\mathrm{b}=1.60\left(\frac{\mathrm{PR}}{\mathrm{E}^{*}}\right)^{(1 / 2)}
$$

where the composite modulus is calculated by equation (2). Otherwise, if the contacting materials present similar elastic properties, the parameter $b$ is calculated by

$$
\mathrm{b}=2.15\left(\frac{\mathrm{PR}}{\mathrm{E}}\right)^{(1 / 2)}
$$

where $E$ is the Young modulus for the material for both cylinders. In equations (4) and ( $4 a$ ), parameter $R$ represents the relative curvature of contact and is given by

$$
\mathrm{R}=\frac{\mathrm{R}_{\mathrm{i}} \mathrm{R}_{\mathrm{j}}}{\left(\mathrm{R}_{\mathrm{i}} \pm \mathrm{R}_{\mathrm{j}}\right)}=\frac{\mathrm{R}_{\mathrm{i}} \mathrm{R}_{\mathrm{j}}}{\Delta \mathrm{R}}
$$


where the \pm sign depends on the curvature of the contacting surfaces, i.e., depends on the contact geometry being internal(-) or external (+). Thus, equation (3) can be rewritten in a form similar to that suggested by Johnson [5], i.e.,

$$
\delta=\frac{\mathrm{P}}{\pi \mathrm{E}^{*}}\left\{\frac{2}{3}+\ln \left(\frac{8 \Delta \mathrm{RE}}{\mathrm{c}^{2} \mathrm{P}}\right)\right\}
$$

in which parameter $E^{\prime \prime}$ corresponds to $E^{*}$ or $E$ and $c$ assumes the value 2.15 or 1.60 depending if the material properties of colliding cylinders are similar or not, respectively.

\subsection{Goldsmith Model}

Also based on the Hertz theory, Goldsmith [13] proposed an expression for indentation as a function of the contact force, $P$, suitable for the internal impact between a shaft inside a cylinder. The Goldsmith model is written as,

$$
\delta=P\left(\frac{\sigma_{i}+\sigma_{j}}{1}\right)\left[\ln \left(\frac{1^{m}}{\operatorname{PR}\left(\sigma_{i}+\sigma_{j}\right)}\right)+1\right]
$$

where $\sigma_{\mathrm{k}}$ is a material parameter, evaluated by,

$$
\sigma_{\mathrm{k}}=\frac{1-v_{\mathrm{k}}^{2}}{\mathrm{E}_{\mathrm{k}}}, \quad(\mathrm{k}=\mathrm{i}, \mathrm{j})
$$

$R$ is the relative curvature as represented in equation (5) and applied to the internal contact geometry, $l$ is the axial length of the cylinder and the exponent $m$ is unitary. In equation (7a) the quantities $v_{k}$ and $E_{k}$ are the Poisson's ratio and the Young's modulus associated with the material of each cylinder. Since the exponent $m=1$ considered by Goldsmith leads to a problem of inconsistency of units in expression (7), Dubowsky and Freudenstein [14] proposed a similar expression with an exponent $m=3$. A comparative study between these two expressions is carried out by Flores et al. [29], in which the solution corresponding to the time variation of indentation, the normal contact force and the force-indentation ratio presented by these two models are discussed.

Considering that the contact force as a force per unit length, and assuming similar elastic properties for the contacting cylinders, the contact model proposed by Goldsmith and expressed by equation (7) can be reformulated, resulting in 


$$
\delta=\frac{\mathrm{P}}{\pi \mathrm{E}^{*}}\left\{\ln \left(\frac{\pi \mathrm{E}^{*}}{\mathrm{PR}}\right)+1\right\}
$$

Initially the Goldsmith model has been derived for internal contact geometry. However, equation (8) can be applied to internal and external contact geometry, changing the sign in equation (5), which quantifies the relative curvature of colliding cylinders.

All the relations between force and indentation defined by the models presented here, for the contact between cylindrical geometries, use a logarithmic function. This is not surprising, since all models are derived from the Hertz contact theory. This function imposes some mathematical and physical limitations on the models, particularly for internal contact geometries with lower clearance values, i.e. for conformal contact conditions. This aspect is presented and discussed in detail in Section 3 of this work.

\subsection{Lankarani and Nikravesh Model}

The simplicity of the model suggested by Lankarani and Nikravesh [10] for implementation in a computational program stems from the fact that the contact force can be expressed as an explicit function of the indentation. Furthermore, this model accounts for the energy dissipation during the impact process, being selected by many researchers to represent the contact/impact phenomena. However, for comparison to all the other models considered in this work, which are purely elastic, the term accounting for the energy dissipated during impact is neglected. Thus, the model suggested by Lankarani and Nikravesh can be expressed in terms of indentation and written as

$$
\delta=\left\{\frac{3 P}{4 \pi E^{*} R^{(1 / 2)}}\right\}^{(1 / n)}
$$

being $E^{*}$ and $R$ evaluated using equations (2a) and (5), respectively. Equation (9) can be also used for internal and external contact geometries, depending on the sign taken in equation (5). The value for the exponent $n$ is equal to 1.5, for contact between spherical bodies. In this case equation (9) represents the contact law proposed by Hertz. Thus, assuming a uniform force distribution over the length of the cylinders and neglecting boundary effects, Hunt and Crossley [8] suggested the use of equation (9) with an 
exponent $n$ in the range of 1.0 to 1.5 , extending in this form the use of the original Hertz model to handle cylindrical contact.

\section{Validity Domains of Analytical Cylindrical Models}

The models proposed by Johnson, Radzimovsky and Goldsmith represent the indentation as a function of contact force, $P$, in a logarithmic form. From a physical point of view, the functions expressed in equations (1), (6) and (8) should exhibit a continuous monotonically increasing behavior, i.e. the values obtained for the indentation must be always positive and must increase with increasing load. This trend is always observed for external contacting cylinders, but not for internal contact or loads beyond certain values. For example, considering Johnson's contact model with a clearance value $5 \mu \mathrm{m}$, for load values higher than approximately $1 \mathrm{kN} / \mathrm{mm}$, the indentation value decreases with increasing load, which is physically inconsistent, as illustrated in Figure 2.

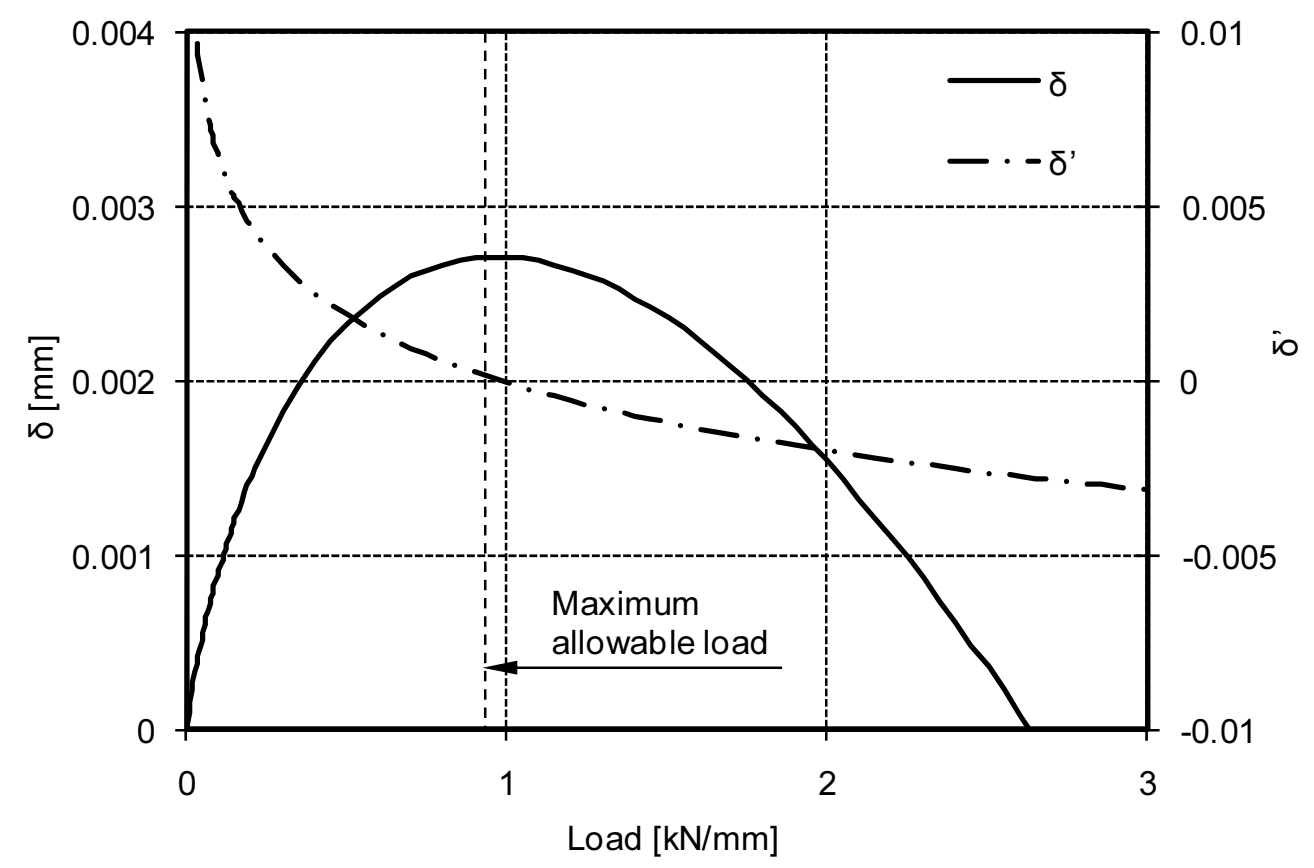

Figure 2 - Indentation and its derivate function for Johnson's contact model for internal cylindrical contact with a clearance of $5 \mu \mathrm{m}$.

This behavior is well characterized by the curve that corresponds to the indentation derivate function. In fact, the derivate function presents a continuous monotonically 
decreasing behavior, reaching zero. This corresponds to the maximum value of the indentation function, beyond which it takes negative values. Thus, for internal contact it is necessary to define the validity domains of the models, which depend on the clearance value and on the material properties.

\subsection{Specification of Validity Domains}

In order to guarantee that the mathematical and physical requirements are satisfied, the logarithmic function of equation (1), which represents Johnson's contact model and consequently the ESDU - 78035 model, must be equal to or greater than 2 . This leads to a load limit value for each clearance value given by

$$
\ln \left(\frac{4 \pi \mathrm{E}^{*} \Delta \mathrm{R}}{\mathrm{P}}\right) \geq 2 \rightarrow \mathrm{P}_{\text {lim }} \leq \frac{4 \pi \mathrm{E}^{*} \Delta \mathrm{R}}{e^{2}}
$$

Just like the Johnson model, in internal contact, the validity of the Radzimovsky model also depends on the clearance values and on the elastic properties of the materials. For stiff materials in which both cylinders have similar Poisson's coefficient and Young's modulus values, the Radzimovsky model is valid for loads lower than the value given by

$$
\ln \left(\frac{8 \mathrm{E} \Delta \mathrm{R}}{2.15^{2} \mathrm{P}}\right) \geq 0 \rightarrow \mathrm{P}_{\lim } \leq \frac{8 \mathrm{E} \Delta \mathrm{R}}{2.15^{2}}
$$

For cylinders with materials having different elastic properties, the equation that defines the validity domain is similar, but $E$ is replaced by the composite modulus $E^{*}$ and the constant 2.15 becomes 1.60 . The Goldsmith model has also a specific validity domain, which depends not only on the material properties and clearance values but also on the dimensions of the bodies. The load limit for the Goldsmith model is

$$
\ln \left(\frac{\pi \mathrm{E}^{*}}{\mathrm{PR}}\right) \geq 0 \rightarrow \mathrm{P}_{\lim } \leq \frac{\pi \mathrm{E}^{*}}{\mathrm{R}}
$$

Contrary to the Johnson and the Radzimovsky models, the contact geometry is not handled by the Goldsmith model in the same way. In this model, the indentation is not a function of $\Delta R$ but, instead, depends on the relative curvature $R$ defined by equation (5). In order to define its validity domain as a load function of clearance value, as done for the others models, it is necessary to reconsider equation (5). For internal contact, the clearance 
is defined as the difference between the radii of colliding cylinders. If the clearance value is very low compared to the radius of the larger body, it can be neglected, so that the quantity $\left(R_{i}-\Delta R\right)$ can be taken as equal to $R_{i}$, this is

$$
\mathrm{R}=\frac{\mathrm{R}_{\mathrm{i}} \mathrm{R}_{\mathrm{j}}}{\left(\mathrm{R}_{\mathrm{i}} \pm \mathrm{R}_{\mathrm{j}}\right)}=\frac{\mathrm{R}_{\mathrm{i}} \mathrm{R}_{\mathrm{j}}}{\Delta \mathrm{R}}=\frac{\mathrm{R}_{\mathrm{i}}\left(\mathrm{R}_{\mathrm{i}}-\Delta \mathrm{R}\right)}{\Delta \mathrm{R}} \simeq \frac{\mathrm{R}_{\mathrm{i}}{ }^{2}}{\Delta \mathrm{R}}
$$

Substituting the value of equation (13) into equation (12), the limit load value becomes an explicit function of the dimensions of the bodies

$$
\mathrm{P}_{\lim } \leq \frac{\pi \mathrm{E}^{*} \Delta \mathrm{R}}{\mathrm{R}_{\mathrm{i}}^{2}}
$$

The validity domain of the Johnson, Radzimovsky and Goldsmith models for differences between the cylinder diameters varying from $5 \mu \mathrm{m}$ to $10 \mathrm{~mm}$ is shown in Figure 3. Note that the lower values of this range are associated to clearances in typical mechanical systems while the higher values are associated with other types of cylindrical contact. Since the validity domain also depends on the material properties, Figure 3 illustrates the domain for a stiff material, such as steel in which $E=207 \mathrm{GPa} ; v=0.3$, with fill symbols, while blank symbols represent the behavior of a soft material, e.g. aluminum for which $E=70 \mathrm{GPa} ; v=0.35$. It can be concluded that for very small clearance values, the validity domain is very restricted, being this limitation even more pronounced for soft materials. As expected, the validity domain decreases for soft materials and for smaller clearances. For example, with a clearance of $5 \mu \mathrm{m}$ the limit load for the Johnson's model is $967 \mathrm{~N} / \mathrm{mm}$ for steel and only $339 \mathrm{~N} / \mathrm{mm}$ for aluminum. Thus, particularly for internal contact with very small clearance values, special care must be taken with the application of the Johnson model as it may not be representative of the contact indentation relation.

Comparing the Radzimovsky and Johnson models for materials with similar elastic properties and for the whole set of clearance values, it is observed that the validity domain of the Radzimovsky model is $46 \%$ to $44 \%$ higher than the Johnson model, for stiff and soft materials, respectively. 


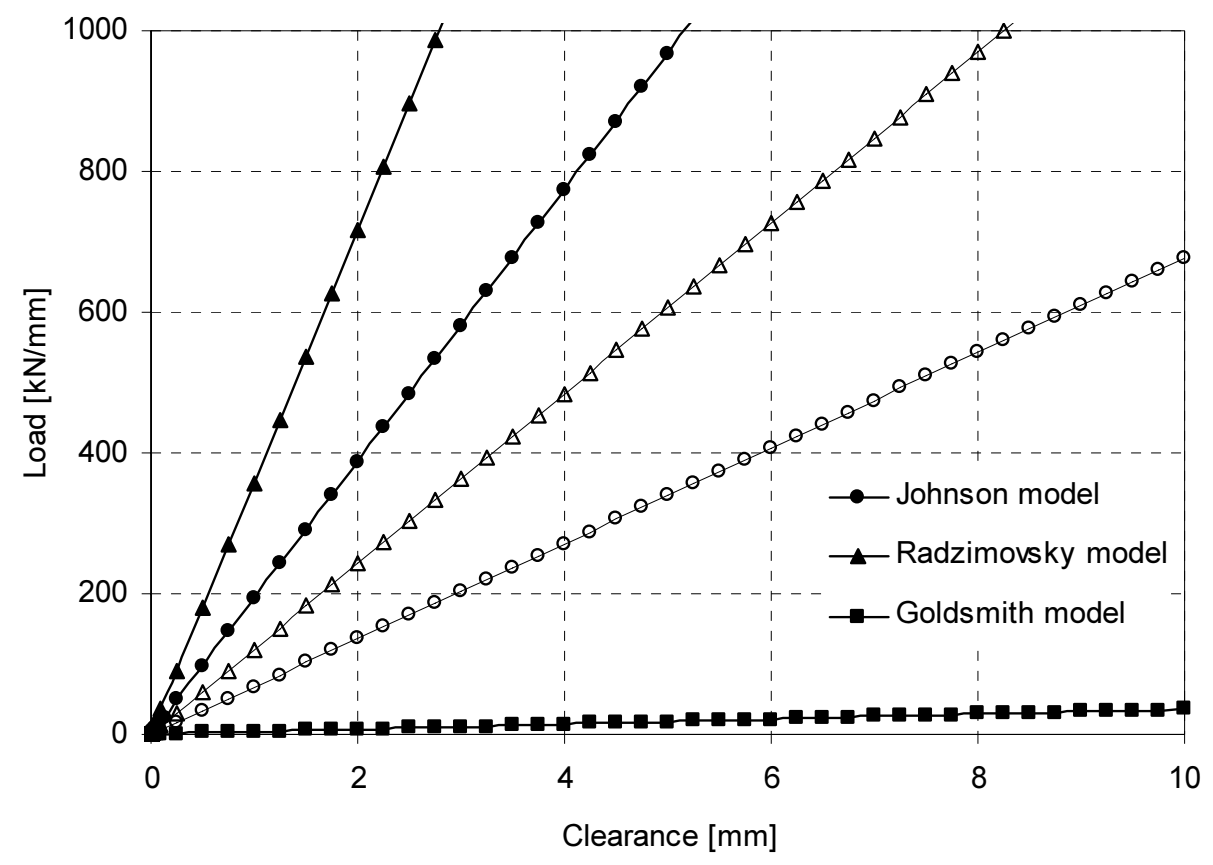

Figure 3 - Validity domain for the different cylindrical contact force models. The filled symbols are for a stiff materials with elastic properties of $E=207 \mathrm{GPa}$ and $v=0.3$ while blank filled symbols are for soft materials with $E=70 \mathrm{GPa}$ and $v=0.35$.

It is worth noting that the Goldsmith model, considering an $R_{i}$ value of $10 \mathrm{~mm}$ for stiff materials has a very limited validity range, as observed in Figure 3. Comparing the three models, it can be concluded that the Goldsmith model presents the narrowest validity domain. Furthermore, this validity domain does not only depend on the clearance value and materials' properties, as it is also strongly dependent on the dimensions of the bodies in contact. This trend is illustrated in Figure 4, which represents the validity domain as a function of clearance values, in the range of $5 \mu \mathrm{m}$ to $10 \mathrm{~mm}$, and dimensions of bodies $R_{i}$ with values in the range 1 to $300 \mathrm{~mm}$. For the same clearance, the limit load varies strongly as a function of $R_{i}$. However, increasing $R_{i}$ reduces the value of limit load and consequently decreases the validity domain of the Goldsmith model. In fact, according to equation (14) for very low clearance values the limit load is reached quickly, even for low $R_{i}$ values. For the Johnson and the Radzimovsky models the validity domain is also reduced with the increasing softness of the materials.

In summation, it is concluded that all models considered have similar expressions, even though the contact geometric is not accounted for in the same way, and each model has a specific validity domain in the case of internal contact. By using the physical considerations expressed before the Lankarani and Nikravesh model, represented by 
equation (9) is valid in the complete domain, since equation does not include a logarithmic term and so does not present mathematical limitations. For external contacting cylinders, all functions of all models are continuous and monotonically increasing, and for all binomials $\Delta R /$ load, the indentation value is always positive. Therefore, for external contact all models overviewed are valid representations of the indentation-contact force relation.

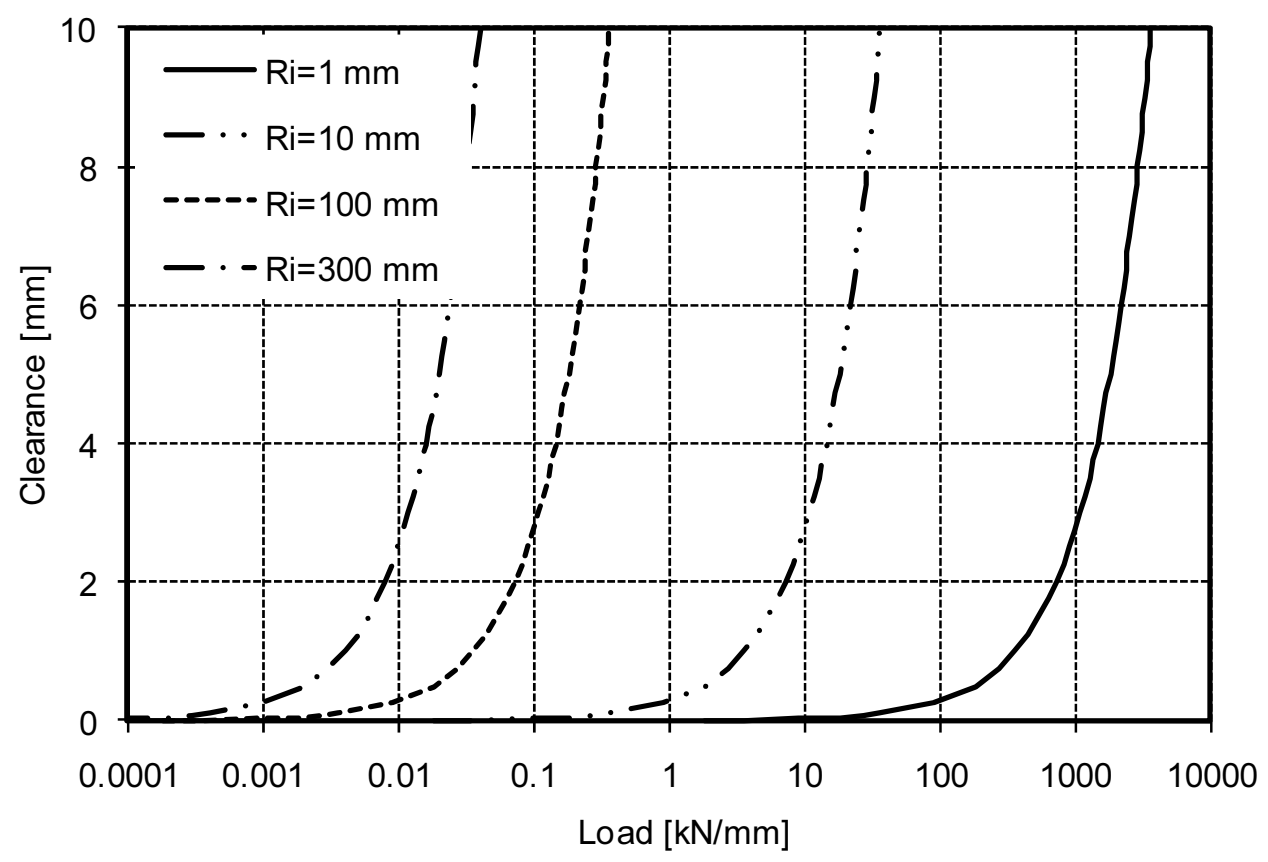

Figure 4 - Validity domain for the Goldsmith model as a function of the dimensions of the bodies in contact for material properties of $E=207 \mathrm{GPa}$ and $v=0.3$.

\subsection{Comparative Study of the Different Models}

A comparative study to evaluate the performance of the models described above in relation to the model presented by Johnson, taken here as the reference, is presented next. The model by Johnson [5] is one of the most popular to establish the relationship between the indentation and the contact force, being the basis to derive the expression proposed by ESDU 78035 Tribology Series [11] for journal bearing applications.

Since the contact between cylinders leads to a wide range of curvature radii the problems of internal and external geometries are analyzed separately. The difference between two given models is 


$$
\text { Error for X model }=\frac{\text { Result applying X model }- \text { Result applying Johnson model }}{\text { Result applying Johnson model }}
$$

A mapping of the differences of the different models, with respect to the Johnson contact model, is presented in Figures 5 through 8, for internal contact, and Figures 9 trough 12, for external contact.

\subsubsection{Internal contact}

In the case of internal contact clearances in the range of $5 \mu \mathrm{m}$ to $10 \mathrm{~mm}$ and loads in the range of 1 to $1000 \mathrm{~N} / \mathrm{mm}$ are tested. Figures 5 through to 8 show the differences of the contact models in relation to the Johnson model. In these figures, and all of the same type that follow, not only particular isolines of differences are identified but also the typical difference between the models observed in particular regions are noted. The limit load value of $1000 \mathrm{~N} / \mathrm{mm}$ is selected according to the validity domain previously identified for each model under the present conditions. For all models, except for the Goldsmith model, loads lower than $1000 \mathrm{~N} / \mathrm{mm}$ are definitely within the validity domain. The Goldsmith model has a very restricted validity domain, especially for lower clearances, as demonstrated. For an $R_{i}=15 \mathrm{~mm}$ the limit loads are $10,15,40,80,120,160,400,800$ $\mathrm{N} / \mathrm{mm}$ for clearances of 5 and 7.5, 10, 25, 50, 75, 100, 250 and $500 \mu \mathrm{m}$, respectively. For clearances above $750 \mu \mathrm{m}$, the Goldsmith model is correctly defined for all loads considered in internal contact. This validity domain is represented by the area above the black dashed line in Figure 6.

The Radzimovsky model differences, with respect to the Johnson model are shown in Figure 5. Differences lower than 5\% are obtained for clearances higher than $500 \mu \mathrm{m}$, while for lower clearances differences equal to or lower than $10 \%$ are obtained. The exception holds for clearances of 5, 7.5, 10 and $25 \mu \mathrm{m}$ and for higher loads where the difference is slightly higher.

The difference of the Goldsmith model, presented in Figure 6, considering only the validity domain for clearance values higher $750 \mu \mathrm{m}$, shows that an excessive value exists in the whole domain. This difference varies varying between $24 \%$, for clearance of $10 \mathrm{~mm}$ and load of $1 \mathrm{~N} / \mathrm{mm}$, to $79 \%$, for a clearance of $750 \mu \mathrm{m}$ and a load value of $1000 \mathrm{~N} / \mathrm{mm}$. Difference diminishes with increasing clearances and increases with increasing loads. 


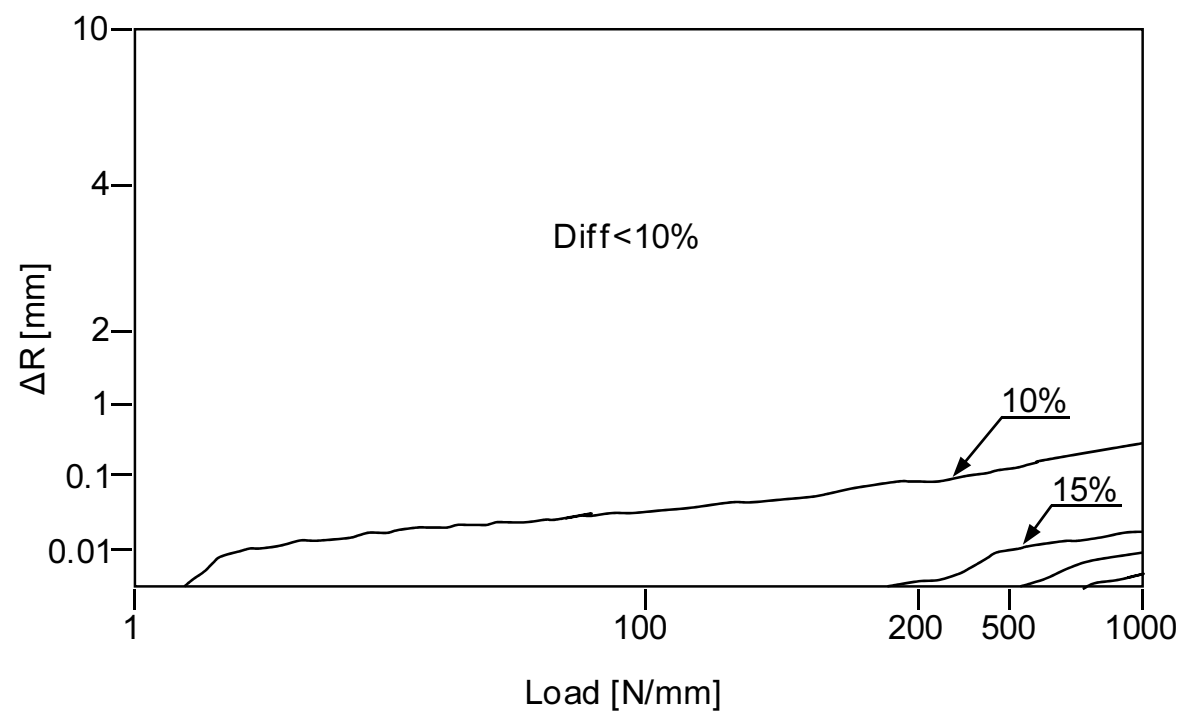

Figure 5: Difference of the Radzimovsky model relative to the Johnson model for internal contact.

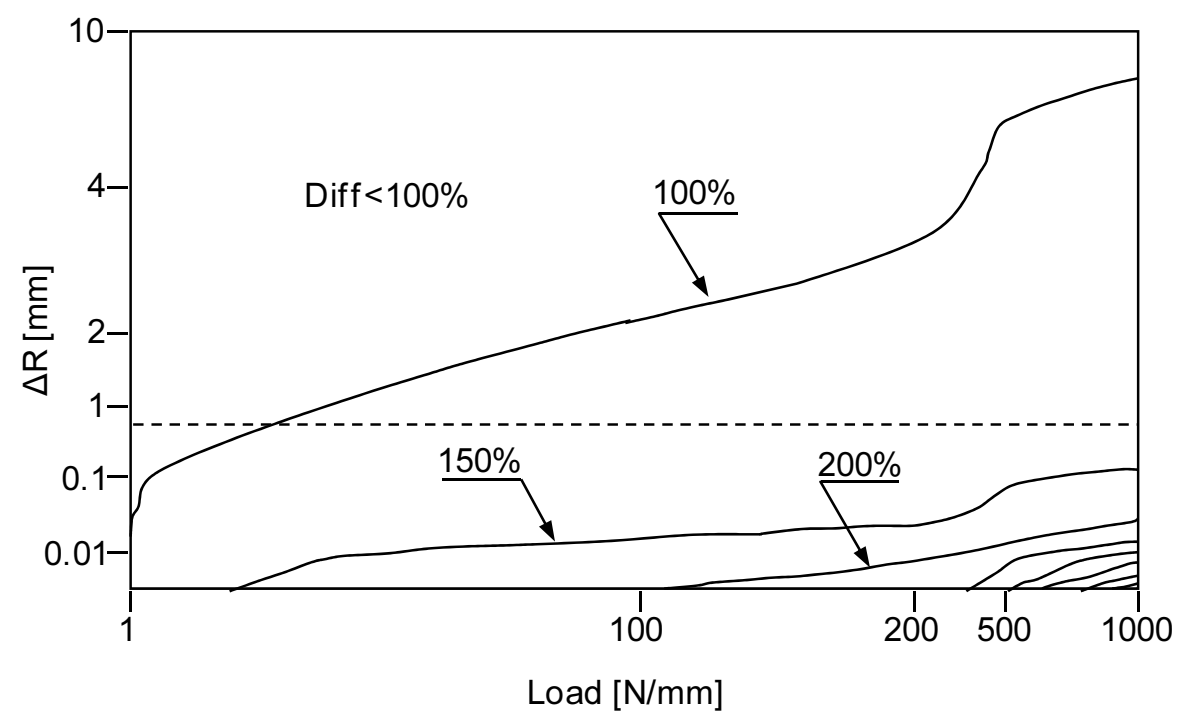

Figure 6: Difference of the Goldsmith model with respect to the Johnson model for internal contact.

The Lankarani and Nikravesh model with an exponent of 1.5 produces maximum differences of $90 \%$ for clearances lower than $100 \mu \mathrm{m}$, as seen in Figure 7. For higher clearance values, the difference decreases. The difference also decreases for lower loads. With an exponent value $n=1.0$ the difference variation is flatter but for the domain of the clearances and loads considered here, higher differences on the vicinity of $100 \%$ are obtained, as observed in Figure 8. 


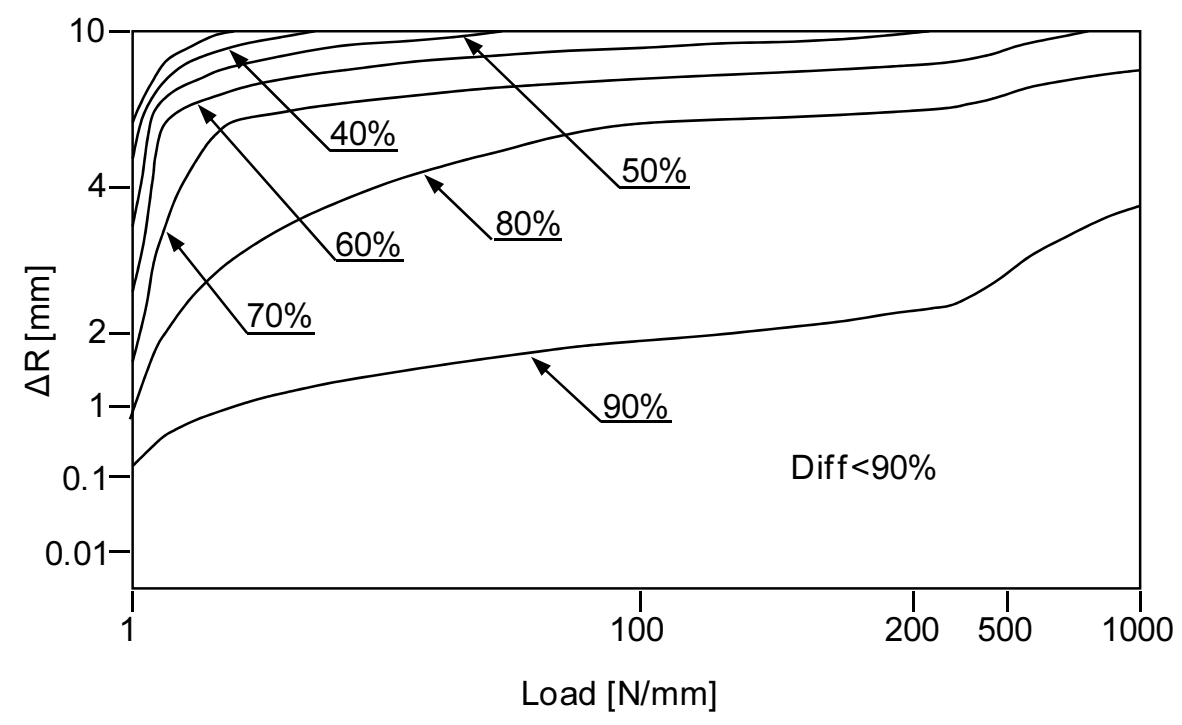

Figure 7: Difference of the Lankarani and Nikravesh model with an exponent value of 1.5 with respect to the Johnson model for internal contact.

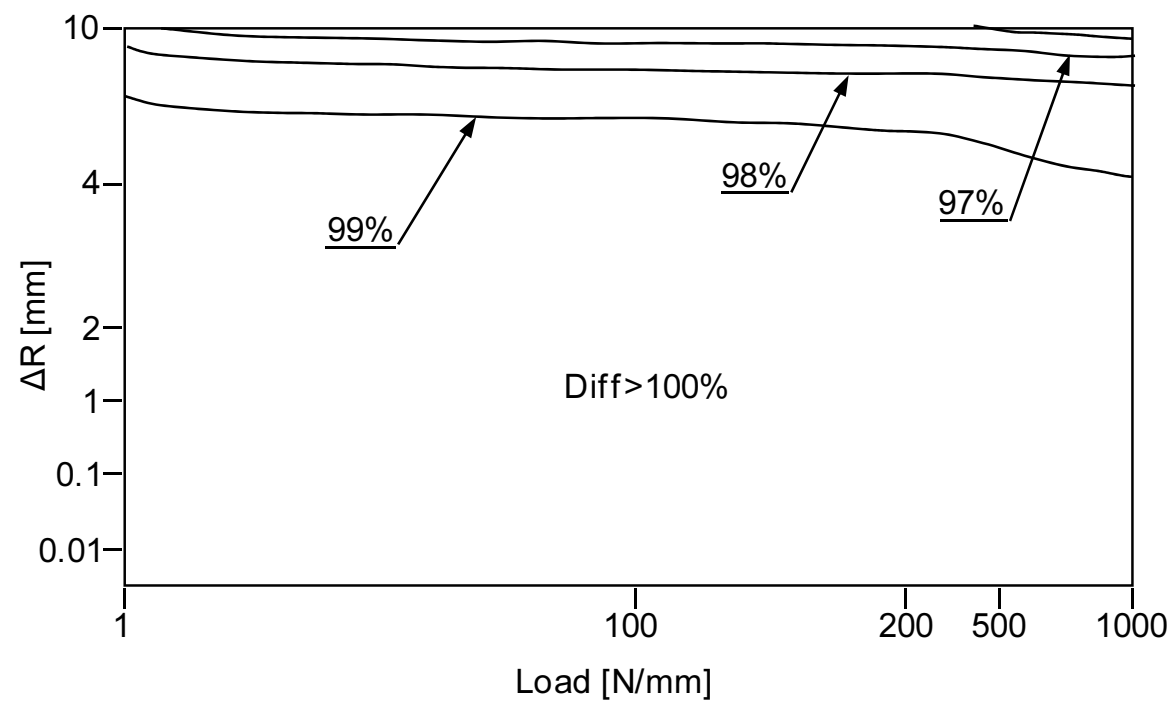

Figure 8: Difference of the Lankarani and Nikravesh model with an exponent value of 1.0 with respect to the Johnson model for internal contact.

Therefore, it is concluded that the Lankarani and Nikravesh model leads to higher differences than the Radzimovsky model, in internal cylindrical contact, regardless of the exponent considered. Thus, like the Goldsmith model, the Lankarani and Nikravesh model is not appropriate for modeling contact between internal cylinders, particularly for lower clearances and higher loads, i.e., in conditions of conformal contact. 


\subsubsection{External contact}

For external cylindrical contact, $\Delta R$ values between 5 and $500 \mathrm{~mm}$ and loads in the range of 1 to $10000 \mathrm{~N} / \mathrm{mm}$ are considered. The same methodology followed for internal contact is used to evaluate the performance of the different contact models for external contact. Figures 9 through 12 illustrate the performance of each model with respect to the Johnson model. The Radzimovsky model presents for the complete domain differences below 3\% with respect to the Johnson model. Only for very low $\left(R_{i}+R_{j}\right)$ and very large loads the Radzimovsky model presents difference in the vicinity of 5\%, as observed in Figure 9.

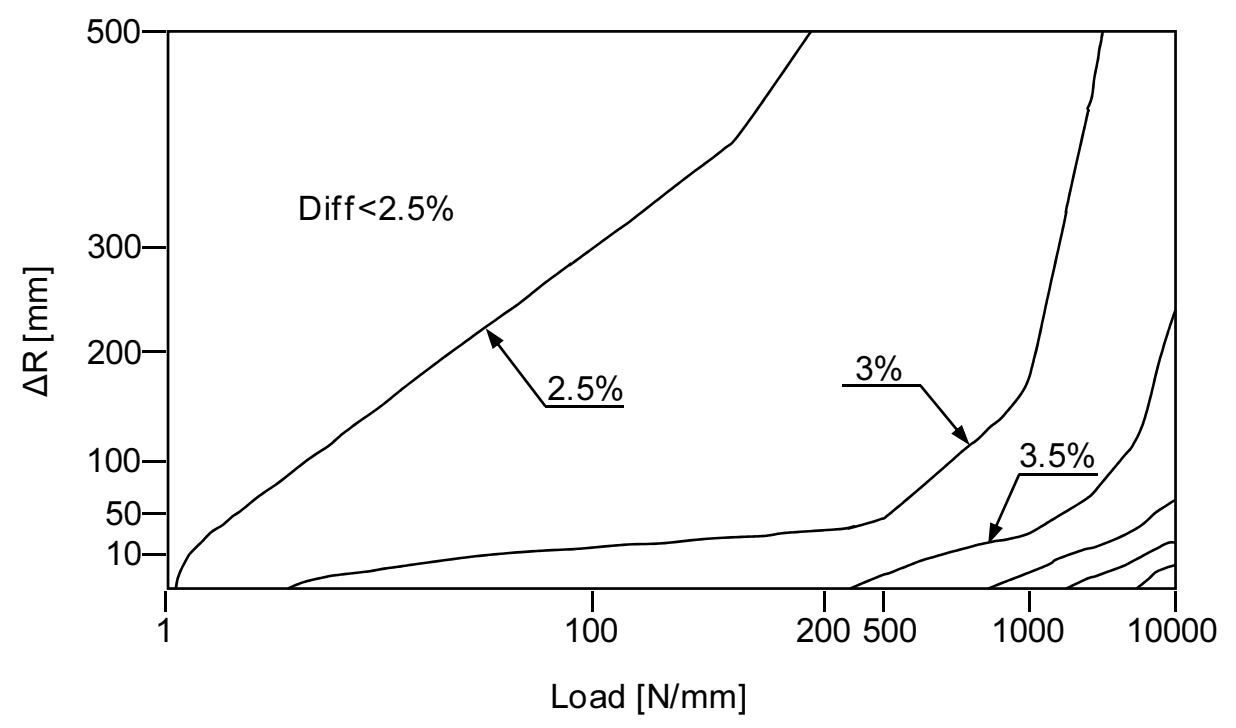

Figure 9: Difference between the Radzimovsky and the Johnson models for external contact.

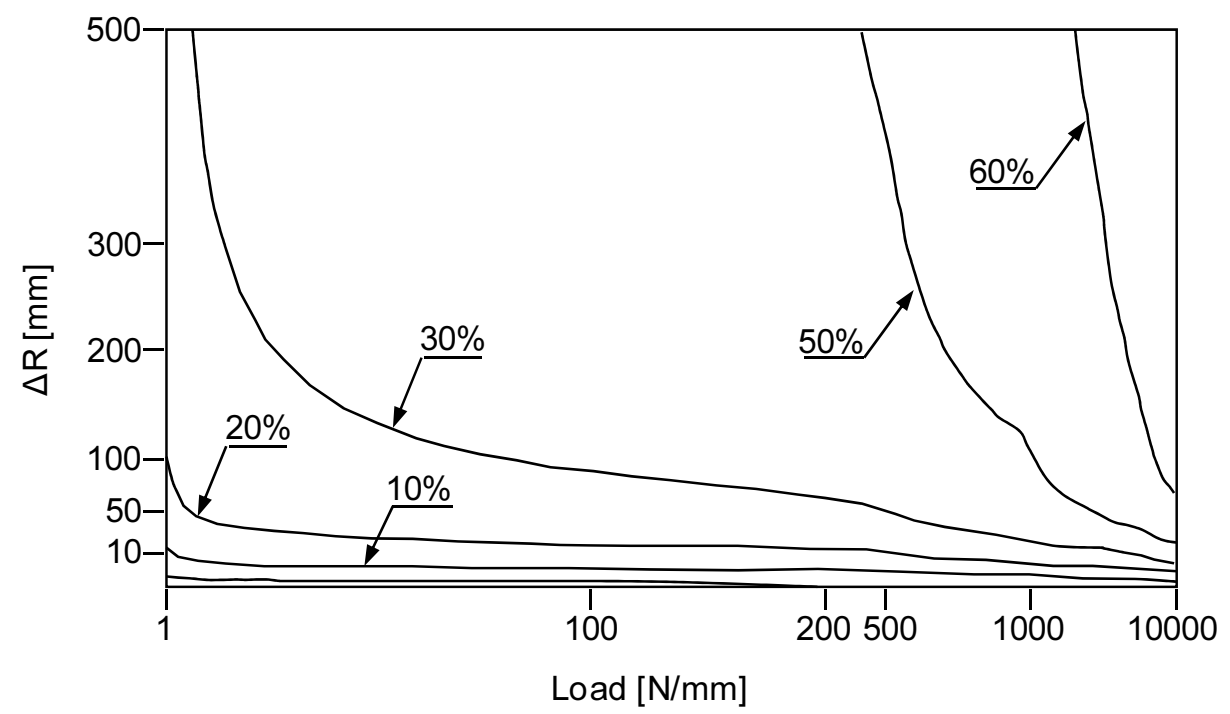

Figure 10: Difference between the Goldsmith and the Johnson models for external contact. 


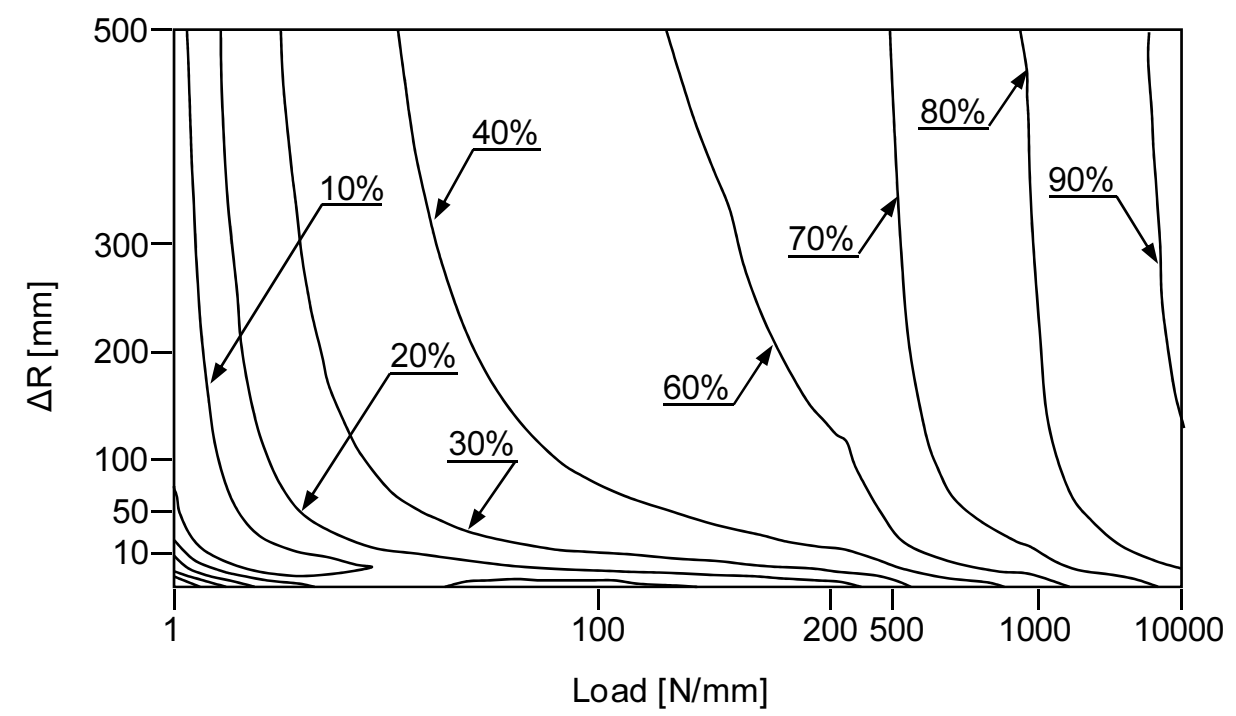

Figure 11: Difference of the Lankarani and Nikravesh model, for an exponent of 1.5, with respect to the Johnson model for external contact.

The differences of the Goldsmith model, illustrated in Figure 10, are smaller than those observed for internal cylindrical contact, especially for the lower $R_{i}+R_{j}$. Nevertheless, the trend of increasing differences with increasing loads remains in the Goldsmith contact model.

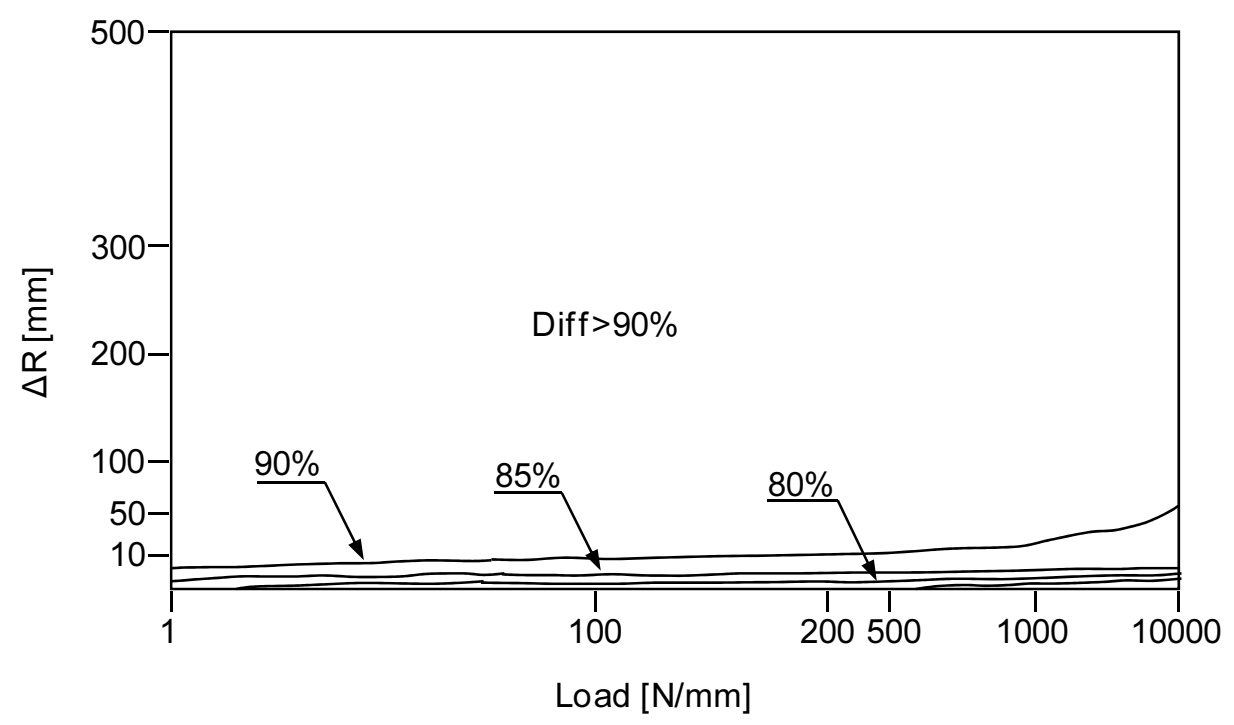

Figure 12: Difference of the Lankarani and Nikravesh model, for an exponent of 1.0, with respect to the Johnson model for external contact.

The trend of the difference growth observed for the Goldsmith model is also evident for the model proposed by Lankarani and Nikravesh with an exponent value of 1.5, depicted 
in Figure 11, being the difference values larger than those of the Goldsmith model. For an exponent of 1.0 the difference is almost constant, as observed also for internal cylindrical contact, even though differences are higher than for $n=1.5$. Figures 11 and 12, show that the Lankarani and Nikravesh model leads to the higher differences than the other models, especially for an exponent of 1.0. This model is, therefore, not appropriate for modeling the contact between external cylinders, regardless of the exponent value considered.

Figures 5 and 6, which represent the constant difference curves versus $\Delta R$ values for internal and external contacting cylinders, respectively, show that for low clearances and forces the Goldsmith and Johnson models present comparable results. Ravn [2] and Flores et al. [29] compared spherical and cylindrical contacts using the Lankarani and Nikravesh, Goldsmith and Dubowsky and Freudenstein models and draw similar conclusions for this range of application of the models. However, for larger values of $\Delta R$ or for higher contact forces, besides the Johnson model taken as reference for this study, only the Radzimovsky model leads to satisfactory accuracy for the two contact geometries. In fact, the models studied here generally exhibit distinct behavior for the two types of contact internal and external. Regarding the Goldsmith model, a rough approximation is obtained, in particular for internal contact. Furthermore, low clearances lead to a negative indentation depth, even for small load values, when these models are applied. This situation is physically impossible, so the validity domain of these models is very limited. Likewise, the model proposed by Lankarani and Nikravesh is found not to be appropriate for modeling contact between cylinders.

\subsection{Clearance and Load Values for Common Mechanisms}

In order to ascertain whether the load limit of each model can restrict its applicability, two common examples of mechanical engineering practice in which internal contacting cylinders are used are analyzed there are the journal bearings and the roller chain drives. The tolerance dimensions for several diameters are established for journal bearings, according to their fit [34]. In this study, a close running fit is selected, which corresponds to an $\mathrm{H8} / \mathrm{f} 7$ adjustment. The corresponding range of clearance values is calculated on the basis of the $\mathrm{H} 8$ and $\mathrm{f} 7$ definitions and their tolerance grades [35]. From a guide for the load capacity of bearings [36], it is possible to define the average tensile strength per unit length

function of the bearing's diameters. Based on a close running fit, Table 1 summarizes the average clearance values and the average tensile strength per unit length of hydrodynamic oil film bearings, for several journal bearing diameters. 
In order to apply the contact models, their mathematical limitations must be known. Equations (1), (6) and (8) establish the limit load values for application of the Johnson, Radzimovsky and Goldsmith models, respectively. These limit load values are presented in Table 1 . The average clearances ranging from $16 \mu \mathrm{m}$ to $83 \mu \mathrm{m}$, which correspond to very low clearance values, and a wide range of load capacities, between $25 \mathrm{~N} / \mathrm{mm}$ and 22381 $\mathrm{N} / \mathrm{mm}$, can be expected for the journal bearing diameters under analysis in general applications. For the roller chain drives three types of roller chain drives are considered: ANSI standard chains $\mathrm{n}^{\circ} .40,80$ and 120 [37]. Based on the average tensile strength and on the distance between internal plates, characteristic of each roller chain type, the average tensile strength per unit length is calculated and presented in Table 2. Clearance values are evaluated according to the tolerance dimensions given by SRAMPORT [38]. A relation between the clearance value of each pin/bushing pair and the pin radii is established and its value multiplied by the pin radii of each standard roller chain drive. The range of clearance values that characterizes the three types of chains is presented in Table 2. The average clearance values and the corresponding limit loads for the Johnson, Radzimovsky and Goldsmith models, for the three types of roller chain drives considered, are also included in Table 2. Clearance values between $22.31 \mu \mathrm{m}$ and $62.50 \mu \mathrm{m}$ and load capacity values ranging from $1498.70 \mathrm{~N} / \mathrm{mm}$ to $4335.02 \mathrm{~N} / \mathrm{mm}$ are obtained for the roller chain drives under study.

Tables 1 and 2 show the values of the pairs clearance-load that are out of the validity domain of each one of the cylindrical models, in terms of the journal bearing diameter and roller chain drive type. In what the journal bearings is concerned, and for the set of average clearance values presented in this study, the Goldsmith model cannot be applied, with the exception of the smallest internal diameter, because all values are out of its validity domain. Therefore, the Goldsmith model must not be used to evaluate contact forces in journal bearings. A similar behavior is observed for roller chain drives, since for the Goldsmith model most values are also out of the validity domain. In the case of journal bearings, the Johnson model has only one value out of the validity domain, which corresponds to higher clearance and higher journal bearing diameter. Regarding the Radzimovsky model, all values are included in the validity domain. For roller chain drives all the values are included in the validity domain of the Johnson and or the Radzimosky models, which suggests that these models are suitable for practical applications of cylindrical contact. Thus, from Tables 1 and 2 it can be concluded that the Goldsmith model should not be used in these practical applications and should be used with caution in other mechanical applications. In addition, this model leads to large differences, even for applications within its validity domain. 


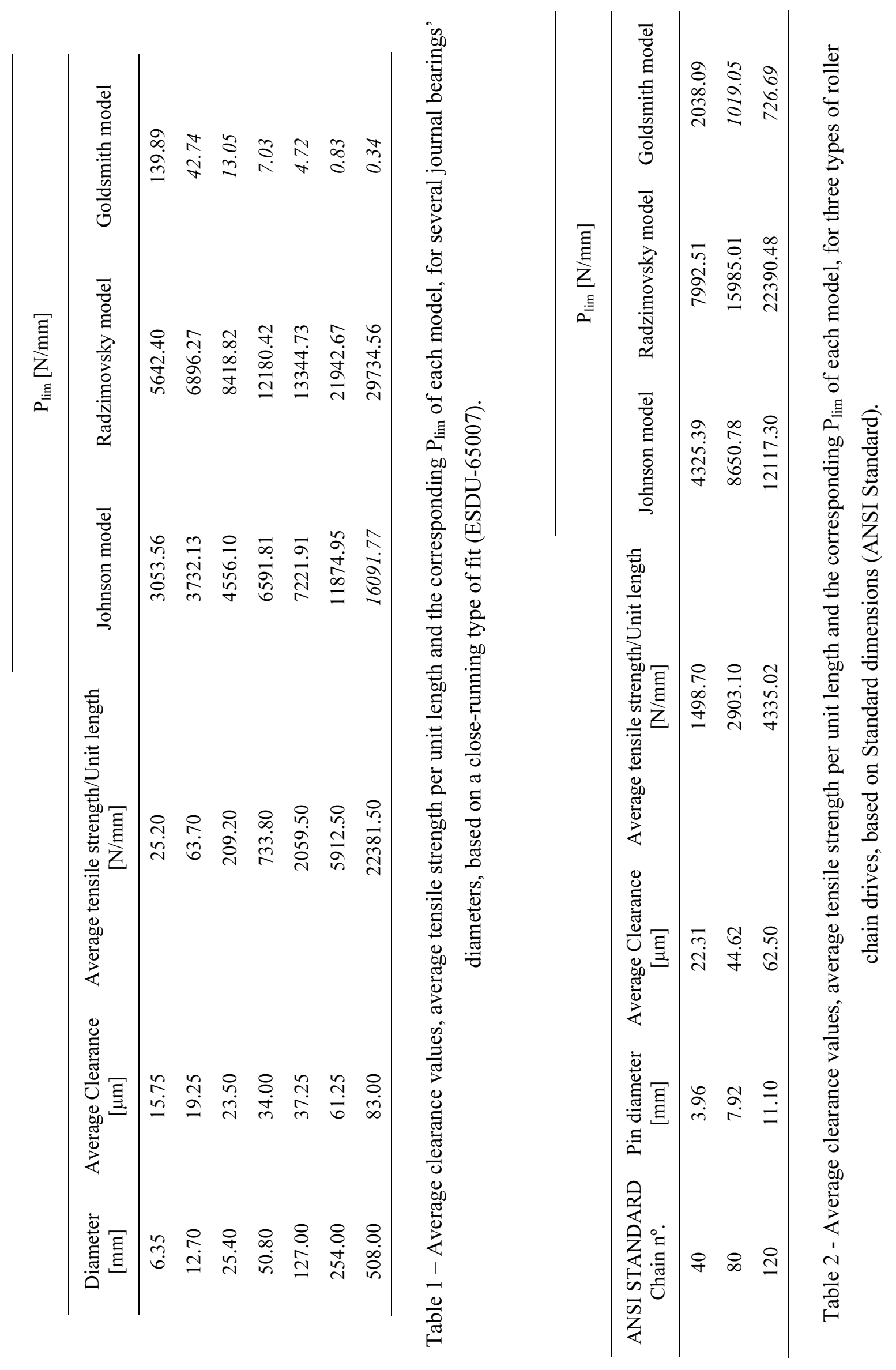




\subsection{Static Analysis of the Different Models}

The analysis of the validity domain of the cylindrical contact models assumes a unitary value for the axial length of the contacting cylinders. Consequently, the influence of the cylinder length on the contact force is not yet taken into account. The contact between cylindrical geometries is in a line and not pontual, and so the contact area assumes a rectangular shape instead of being ellipsoidal. Contrary to spherical contact the cylindrical contact force models is expressed as a load per unit axial length. So it is expected that the variation of the cylinder length affects the behavior exhibited by cylindrical contact models. To evaluate the influence of the cylinder length on the different approaches that describe the contact phenomenon involving bodies with cylindrical geometries a static loading case is performed using a basic journal-bearing geometry. Two different clearance values are analyzed: 0.5 and $0.02 \mathrm{~mm}$. The first is selected because it corresponds to the exaggerated typical clearance of worn equipment, while the second corresponds to the clearance in a typical journal-bearing [36]. Values of 1 and 0.5 are taken for the radius/length ratio to evaluate the influence of the journal-bearing length on the contact force. In this analysis all the models are considered to be purely linear elastic because neither of the models described in the literature that characterize the contact between cylindrical geometries accounts for the energy dissipation that may occur during contact. Spherical and cylindrical models are also used in this study as a form to account to the Lankarani and Nikravesh model, also used for modeling the contact between cylinders, despite being derived for spherical contacting geometries. Figures 13 and 14 illustrate the behavior of the models under study, for clearance values of 0.5 and $0.02 \mathrm{~mm}$ respectively, and for a journal-bearing axial length of $10 \mathrm{~mm}$.

All cylindrical models exhibit a similar behavior, as shown in Figure 13, although small differences in the contact force can be observed for higher penetration values. The Goldsmith model has the smallest contact force values for all the penetration values tested is the exception to this trend. This behavior is not surprising for the Goldsmith contact model, since the generalized stiffness coefficient takes lower values than those obtained with the other cylindrical models.

Comparing the cylindrical models with the spherical contact described by the Lankarani and Nikravesh model, it is observed that in both cases the contact force increases with the penetration depth. Nevertheless, for the same penetration value, smaller contact forces are achieved with the Lankarani and Nikravesh model, which means that the 
contact is softer than with cylindrical models. For very high penetration values, however, identical contact forces are observed for both contact models.

For the $0.02 \mathrm{~mm}$ clearance value, Figure 14 shows that, in general, the behavior of the contact models is similar to that described for a clearance value of $0.5 \mathrm{~mm}$. There are small differences between the cylindrical models, particularly for higher penetration values, being the discrepancy larger for small the clearances, especially between cylindrical and spherical models.

The influence of the contact axial length on the behavior of cylindrical models is illustrated in Figure 15, with respect to the Johnson's model, for a clearance value of 0.02 $\mathrm{mm}$. It is observed that the contact force increases with the axial length of the cylinder. This behavior cannot be predicted by the spherical model, since it does not account for the influence of the length dimension on the contact force. Figure 15 also shows the relative difference values between the Lankarani and Nikravesh and the Johnson models for the two axial lengths considered. Relative differences ranging from $90 \%$ to $40 \%$ are observed between the models for an axial length of $10 \mathrm{~mm}(\mathrm{R} / \mathrm{L}=1.0)$. The maximum difference corresponds to the smallest penetration depth. The same trend is observed for an axial length of $20 \mathrm{~mm}(\mathrm{R} / \mathrm{L}=0.5)$, with difference ranging from $95 \%$ to $70 \%$.

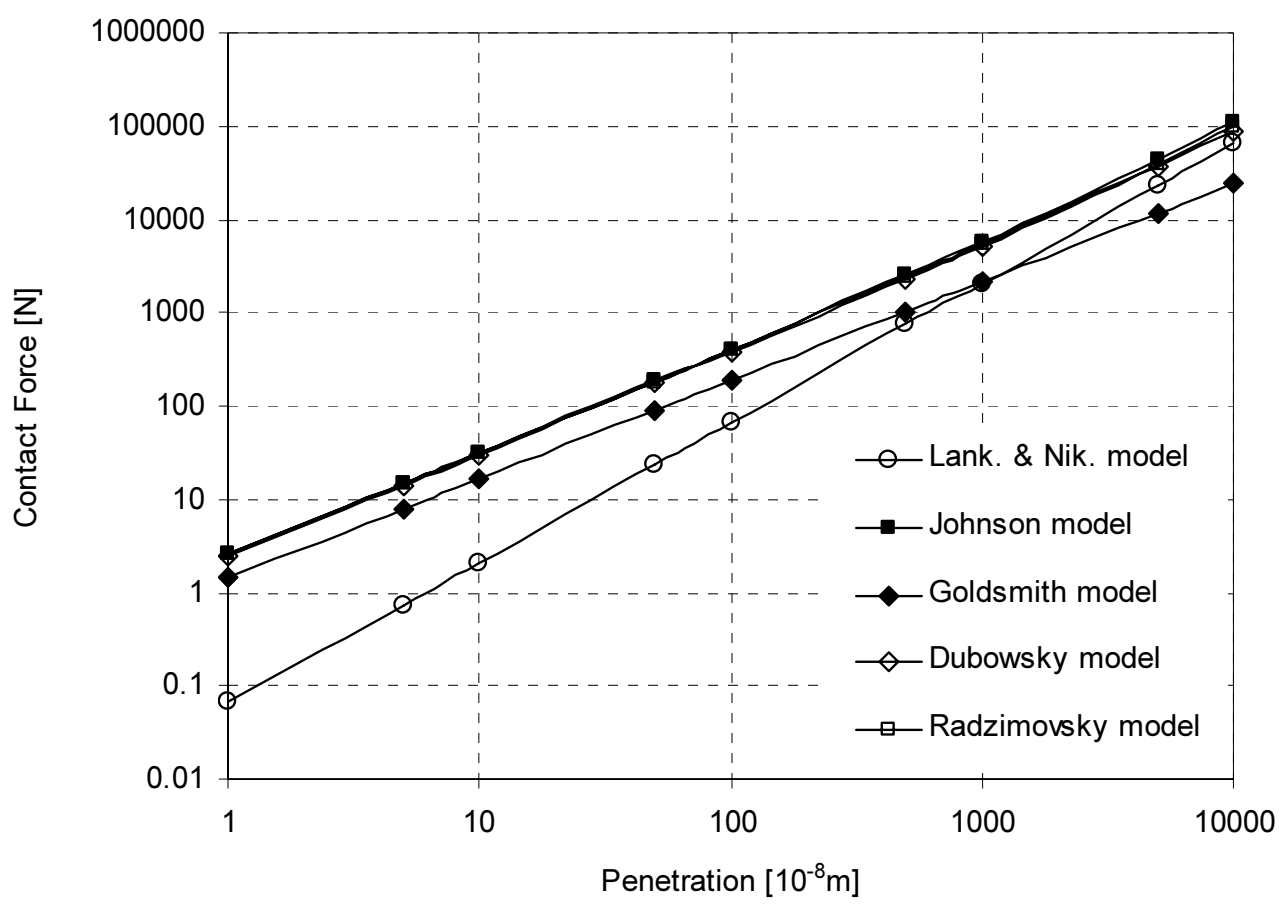

Figure 13 - Contact force vs. penetration depth for a clearance value of $0.5 \mathrm{~mm}$ for cylinders with material properties of $E=207 \mathrm{GPa}$ and $v=0.3$. 


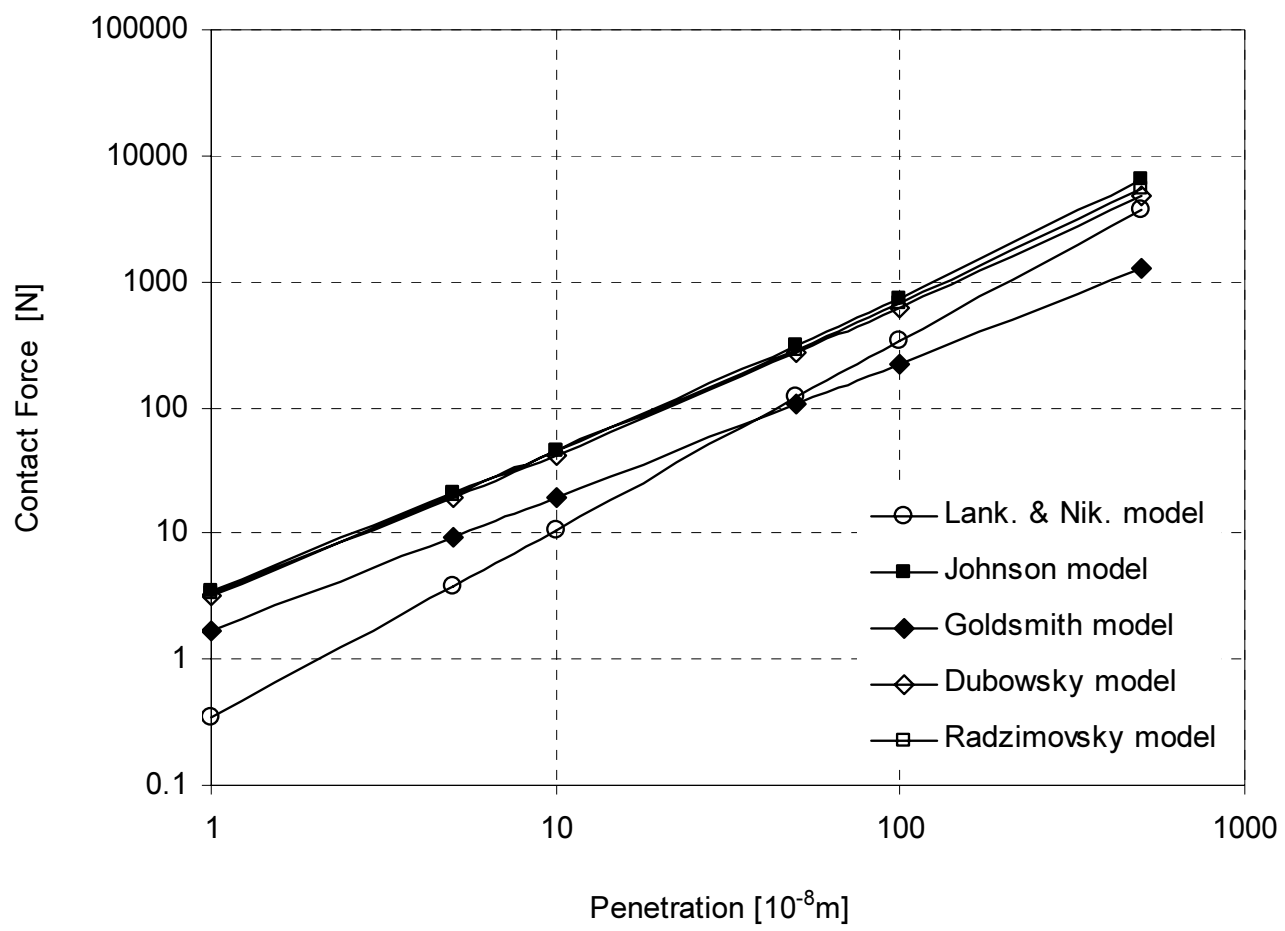

Figure 14 - Contact force vs. penetration depth for a clearance value of $0.02 \mathrm{~mm}$ for cylinders with material properties of $E=207 \mathrm{GPa}$ and $v=0.3$.

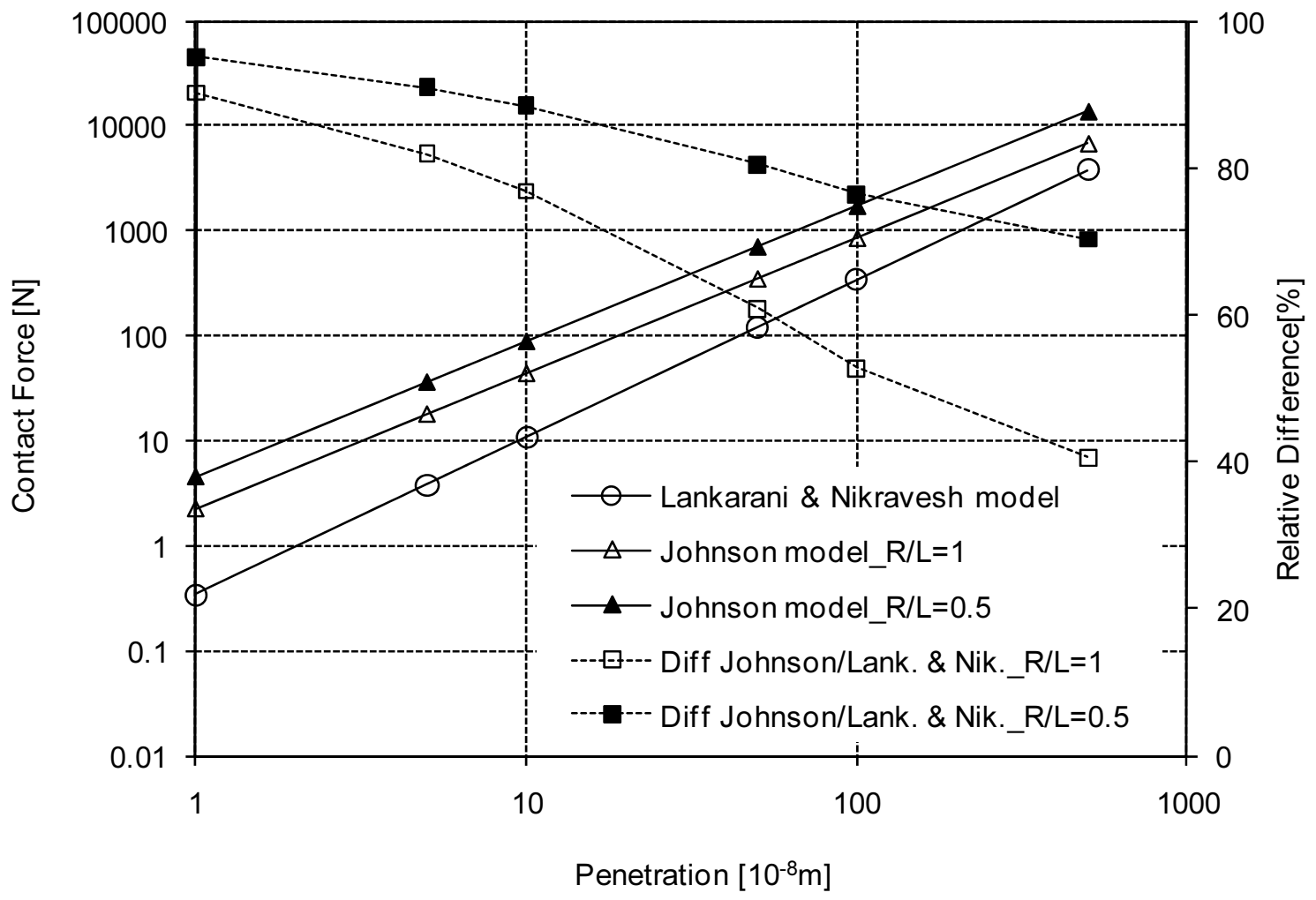

Figure 15 - Influence of cylinder axial length in the behavior presented by the Lankarani and Nikravesh and the Johnson models, for a clearance value of $0.02 \mathrm{~mm}$ and a material with properties $E=207 \mathrm{GPa} ; v=0.3$. 
From Figures 13 through 15 it can be concluded that the Lankarani and Nikravesh model is the least appropriate for modeling the contact between cylindrical geometries. Firstly, it is formulated for spherical contact geometries and therefore the shape and principally the length of the bodies are not taken into account. Secondly, because the contact between cylindrical bodies is stiffer than the contact between spherical bodies, small values of contact force are obtained for the same penetration and this difference is larger with smaller clearances. The Goldsmith model leads to a smaller generalized stiffness coefficient, and consequently to smaller contact force values, when compared to other cylindrical models. This behavior arises from the inconsistency of the units related to the exponent value that affects the axial length of the cylinder. This model should, therefore, not be selected to describe the contact between bodies with cylindrical geometries.

It is also concluded that there are, in general, only small differences between the different cylindrical contact models. Special attention is required in the application of these models, particularly for internal contacting geometries with low clearance values and high penetration depths, since their validity domain depends on the value of the logarithmic function. Therefore, an analytical model free of mathematical and physical limitations, i.e. without domain validity problems, and defining the contact force as an explicit function of penetration can be a useful alternative to modeling the contact between cylindrical geometries.

\section{Conclusions}

Based on the Hertz pressure distribution, different authors have proposed analytical models to study the contact between cylindrical bodies, being some of these models analyzed and discussed in this paper. These cylindrical models have three major shortcomings: $i$ ) they are all iterative if the contact force is to be calculated as a function of indentation, thus requiring the use of less efficient numerical procedures; ii) they have all been proposed as purely elastic models, and are unable to explain the energy dissipation during the impact process; iii) they all include logarithmic functions, which imposes mathematical and physical limitations on their application, particularly for conformal contact conditions with lower clearance values. The exception is the force-penetration relation proposed by Lankarani and Nikravesh, with a modification of the pseudo-stiffness parameter and of the indentation exponent. Due to their mathematical and physical limitations the validity 
domain of each model, which depends on the clearance and material properties, has been identified and discussed.

A comparative assessment of the performance of each model in relation to the Johnson contact model was performed after specifying their validity domains. The results showed that, in general, different models exhibit distinct behavior for both the internal and external contact between cylinders. Furthermore, due to the high differences displayed by all models, it can be concluded that they are not sufficiently accurate for modeling either type of cylindrical contact, apart from the Radzimovsky model. When compared with the Johnson model, the Lankarani and Nikravesh model, for any value of exponent tested, leads to high differences. The Lankarani and Nikravesh contact model, therefore, represents a rough approximation to evaluating the contact between cylindrical bodies, although it relates indentation and contact force by an explicit function. However, the contact force values are underestimated, especially for lower clearance values. Likewise, the study of the Goldsmith model revealed that it also leads to high differences even for applications within its validity domain.

In order to evaluate if the load limit of each model restricts its applicability, two common examples of mechanical engineering practice in which internal contacting cylinders are involved have been analyzed, i.e., a journal bearings and a roller chain drives. This study allows concluding that the Goldsmith model should not be used in the majority of mechanical applications because of its very restricted validity domain. In addition, the study reveals that only the Johnson and the Radzimovsky models are suitable to describe the contact involving colliding cylinders in most of practical applications. Also, when involving the length of the cylinder on the contact forces, the conclusions on the validity and precision of the contact models remain the same, i.e., the use of the Johnson and Radzimovsky contact models are recommended.

\section{References}

[1] Zukas, J.A., Nicholas, T., Greszczuk, L.B., and Curran, D. R., 1982, Impact Dynamics, John Wiley and Sons, New York, New York.

[2] Ravn, P., 1998, Analysis and Synthesis of Planar Mechanical Systems Including Flexibility, Contact and Joint Clearance, Ph.D. Dissertation, DCAMM Report No. S78, Technical University of Denmark, Lyngby, Denmark.

[3] Shivaswamy, S., Lankarani, H.M., 1997, Impact Analysis of Plates Using QuasiStatic Approach, Journal of Mechanical Design, 119, pp. 376-381. 
[4] Johnson K.L., 1982, One hundred years of Hertz contact, Proc. Instn. Mech. Engrs., 196, pp. 363-378.

[5] Johnson, K.L., 1994, Contact Mechanics, Cambridge University Press, Cambridge, England.

[6] Lim C.T. and Stronge, W.J.Stronge, 1999, Oblique elastic-plastic impact between rough cylinders in plane strain, International Journal of Engineering Science, 37, pp. 97-122.

[7] Gugan, D., 2000, Inelastic collision and the Hertz theory of impact, American Journal of Physics, 68(10), pp. 920-924.

[8] Hunt, K.H., and Crossley, F.R., 1975, Coefficient of restitution interpreted as damping in vibroimpact, Journal of Applied Mechanics, 7, pp. 440-445.

[9] Lankarani, H.M., and Nikravesh, P.E., 1990, A Contact Force Model With Hysteresis Damping for Impact Analysis of Multibody Systems, Journal of Mechanical Design, 112, pp. 369-376.

[10] Lankarani, H.M., and Nikravesh, P.E., 1994, Continuous Contact Force Models for Impact Analysis in Multibody Systems, Nonlinear Dynamics, 5, pp. 193-207.

[11] ESDU-78035 Tribology Series, 1978, Contact Phenomena. I: stresses, deflections and contact dimensions for normally-loaded unlubricated elastic components, Engineering Sciences Data Unit, London, England.

[12] Radzimovsky, E. I., 1953, Stress distribution and strength conditions of two rolling cylinders pressed together, Eng. Exp. Sta. Univ. Ill., Bull. 408.

[13] Goldsmith, W., 1960, Impact, 'The Theory and Physical Behaviour of Colliding Solids', Edward Arnold Ltd, London, England.

[14] Dubowsky, S., and Freudenstein, F., 1971, Dynamic Analysis of Mechanical Systems with Clearances, Part 1: Formulation of Dynamic Model, Journal of Engineering for Industry, Series B, 93(1), pp. 305-309.

[15] Ravn, P., Shivaswamy S., Alshaer B. J., Lankarani Hamid M., 2000, Joint clearances with lubricated long bearings in multibody mechanical systems, Journal of Mechanical Design, 122, pp. 484-488.

[16] Lankarani, H.M., Nikravesh, P.E., 1992, Canonical Impulse-Momentum Equations for Impact Analysis of Multibody Systems, Journal of Mechanical Design, 114, pp. 180-186. 
[17] Ahmed, S., Lankarani, H.M., Pereira, M., 1999, Frictional Impact Analysis in OpenLoop Multibody Mechanical Systems, Journal of Mechanical Design, 121, pp.119127.

[18] Lankarani, H.M., 2000, A Poisson-Based Formulation for Frictional Impact Analysis of Multibody Mechanical Systems with Open or Closed Kinematic Chains, Journal of Mechanical Design, 122, pp. 489-497.

[19] Flores, P., Ambrósio, J., 2010, On the Contact Detection for Contact-Impact Analysis in Multibody Systems, Multibody System Dynamics, 24(1), pp. 103-122.

[20] Liu, C.-S., Zhang, K. and Yang, L., 2006, Normal force-displacement relationship of spherical joints with clearances, Journal of Computational and Nonlinear Dynamics, 1, pp. 160-167.

[21] Liu, C.-S., Zhang, K. and Yang, L., 2007, The FEM analysis and approximate model for cylindrical joints with clearances, Mechanism and Machine Theory, pp. 183-197.

[22] Flores, P., Ambrósio, J., Claro, J.C.P., Lankarani, H.M. and Koshy, C.S., 2009, Lubricated revolute joints in rigid multibody systems, Nonlinear Dynamics, 56, pp. 277-295.

[23] Pereira, C., Ambrosio, J., Ramalho, A. and Flores, P., 2010, A methodology for the generation of models for multibody chain drives, Multibody System Dynamics, DOI: 10.1007/s11044-010-9207-X.

[24] Ambrosio, J. and Verissimo, P., 2009, Improved Bushing Models for Vehicle Dynamics, Multibody System Dynamics, 22(4), pp. 341-365.

[25] Ryan, R.R., 1990, ADAMS-Multibody System Analysis Software, Multibody Systems Handbook, Berlin, Springer-Verlag.

[26] Smith, R.C., and Haug, E.J., 1990, DADS-Dynamic Analysis and Design System, Multibody Systems Handbook, Berlin, Springer-Verlag.

[27] Bottasso, C.L., Citelli P., Taldo, A., and Franchi, C.G., 1999, Unilateral Contact Modeling with Adams, In International ADAMS User's Conference, Berlin, Germany, November 17-18, 11p.

[28] Pedersen, S.L., Hansen, J.M. and Ambrósio, J., 2004, a roller chain drive model including contact with guide-bars, Multibody Systems Dynamics, 12(3), pp. 285301.

[29] Flores, P., Ambrósio, J., Claro, J.C.P. and Lankarani, H.M., 2006, Influence of the contact-impact force model on the dynamic response of multi-body systems, Proc. IMechE Part K: Journal of Multi-body Dynamics, 220, pp. 21-34. 
[30] Pedersen, S.L., 2004, Simulation and Analysis of Roller Chain Drive Systems, Ph.D. Dissertation, Department of Mechanical Engineering, Solid Mechanics, Technical University of Denmark, Lyngby, Denmark.

[31] Silva, M., and Ambrósio, J., 2004, Human Motion Analysis Using Multibody Dynamics and Optimization Tools, Technical Report IDMEC/CPM - 2004/001', Instituto Superior Técnico of the Technical University of Lisbon, Lisbon, Portugal.

[32] Hertz, H., 1896, On the contact of solids - On the contact of rigid elastic solids and on hardness (Translated by D. E. Jones and G. A. Schott), Miscellaneous Papers, Macmillan and Co. Ltd., London, England, pp 146-183.

[33] Roark's, 1989, Formulas for Stress \& Strain, McGraw-Hill, $6^{\text {th }}$ Edition.

[34] Shigley, J.E., Mischke, C.R., 1989, Mechanical Engineering Design, Mc Graw Hill, $5^{\text {th }}$ Edition.

[35] Parker, M.A., 1995, Drawing Standards for Computer-aided Engineering, England City and Guilds/Macmillan, $1^{\text {st }}$ Edition.

[36] ESDU - 65007 Tribology Series, 1965, General Guide to the Choice of Journal Bearing Type, Engineering Sciences Data Unit, London, England.

[37] Shigley, J.E., Mischke, C.R., 1990, Power Transmission Elements - A Mechanical Designers' Workbook, Chapter 4 - Chain Drives, by Kurt M. Marshek, Mc Graw Hill, Inc., pp. 159-207.

[38] SRAMPORT - Mechanical Drives Company; Activity: Manufacture of bearings, gears, gearing and driving elements, Coimbra, Portugal. 